\title{
Intensity modulated radiation therapy for breast cancer: current perspectives
}

This article was published in the following Dove Press journal:

Breast Cancer - Targets and Therapy

6 March 2017

Number of times this article has been viewed

\author{
Milly Buwenge' \\ Silvia Cammelli' \\ Ilario Ammendolia' \\ Giorgio Tolento' \\ Alice Zamagni' \\ Alessandra Arcelli ${ }^{2}$ \\ Gabriella Macchia ${ }^{3}$ \\ Francesco Deodato 3 \\ Savino Cilla ${ }^{4}$ \\ Alessio G Morganti' \\ 'Department of Experimental, \\ Diagnostic and Specialty Medicine - \\ DIMES, Radiation Oncology Center, \\ University of Bologna, S.Orsola- \\ Malpighi Hospital, ${ }^{2}$ Radiation \\ Oncology Unit, Bellaria Hospital, \\ Bologna, ${ }^{3}$ Radiation Oncology Unit, \\ ${ }^{4}$ Medical Physics Unit, Research and \\ Care Foundation 'Giovanni Paolo II,' \\ Catholic University of Sacred Heart, \\ Campobasso, Italy
}

Correspondence: Milly Buwenge Department of Experimental, Diagnostic and Specialty Medicine - DIMES, Radiation Oncology Center, University of Bologna, S.Orsola-Malpighi Hospital, via Giuseppe Massarenti 9, 40I38,

Bologna, Italy

Tel $+3905 \mid 6363$ I36

Fax +3905I 6364336

Email mbuwenge@gmail.com
Background: Owing to highly conformed dose distribution, intensity modulated radiation therapy (IMRT) has the potential to improve treatment results of radiotherapy (RT). Postoperative RT is a standard adjuvant treatment in conservative treatment of breast cancer (BC). The aim of this review is to analyze available evidence from randomized controlled trials (RCTs) on IMRT in BC, particularly in terms of reduction of side effects.

Methods: A literature search of the bibliographic database PubMed, from January 1990 through November 2016, was performed. Only RCTs published in English were included.

Results: Ten articles reporting data from 5 RCTs fulfilled the selection criteria and were included in our review. Three out of 5 studies enrolled only selected patients in terms of increased risk of toxicity. Three studies compared IMRT with standard tangential RT. One study compared the results of IMRT in the supine versus the prone position, and one study compared standard treatment with accelerated partial breast IMRT. Three studies reported reduced acute and/or late toxicity using IMRT compared with standard RT. No study reported improved quality of life. Conclusion: IMRT seems able to reduce toxicity in selected patients treated with postoperative RT for BC. Further analyses are needed to better define patients who are candidates for this treatment modality.

Keywords: intensity modulated radiation therapy, breast cancer, literature review

\section{Introduction}

Breast carcinoma (BC) is the most common malignancy in females. ${ }^{1}$ Radiotherapy (RT) is a standard adjuvant therapy both after mastectomy and after conservative breast surgery owing to improved local control and survival. ${ }^{2}$ However, RT can produce acute and late side effects and worsening cosmesis and quality of life (QoL). ${ }^{3}$

Intensity modulated radiation therapy (IMRT) is a modern treatment technique based on delivery of nonuniform fluence and is able to deliver a highly conformed dose to the target. IMRT represents now a standard RT technique in several neoplasms owing to improved dose distribution and reduced dose to organs at risk.

Several planning studies recorded improved dose distribution and better conformity and homogeneity index (Figure 1) with the advantage of simultaneous integrated boost (a technique allowing the irradiation of several targets at different dose levels in the same treatment session yet sparing healthy organs) to tumor bed, even in adjuvant RT of BC. ${ }^{4-6}$ Furthermore, several phase II or retrospective studies on IMRT in BC showed decreased acute radiation-induced toxicity and reduced chronic breast edema with similar results in terms of local control and secondary malignancies compared with standard 


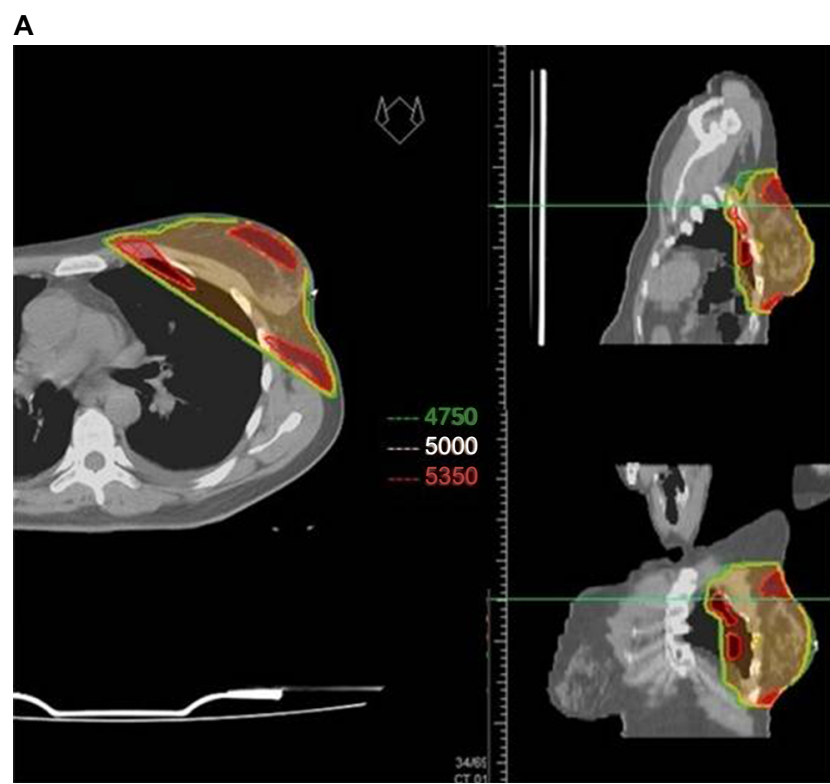

B

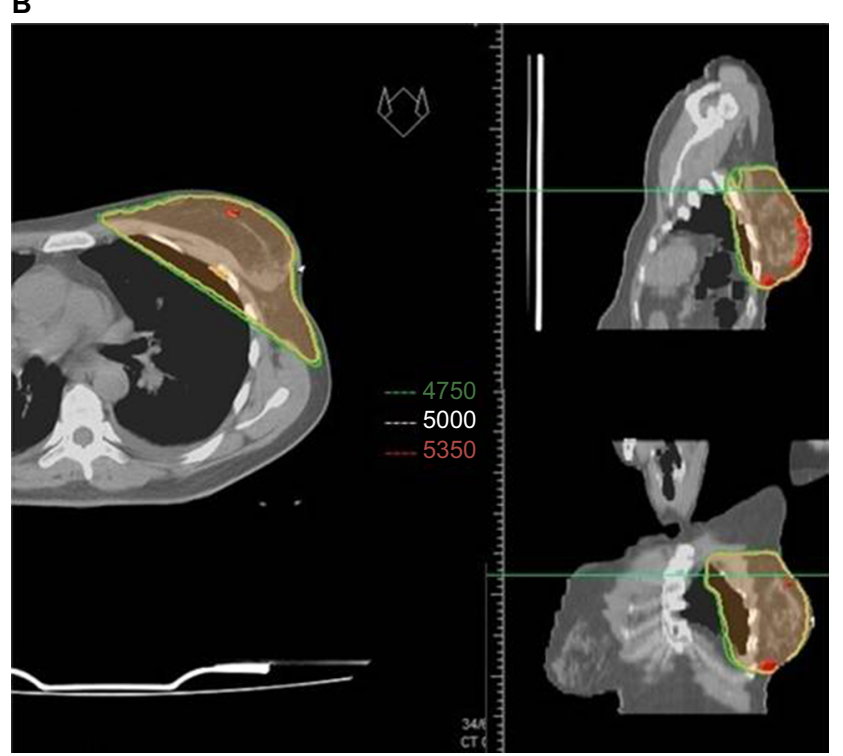

Figure I Comparison of dose distribution between a typical 3D-conformal RT wedged tangential treatment (A) and a tangential IMRT treatment (B).

Abbreviations: 3D, three dimensional; IMRT, intensity modulated radiation therapy; RT, radiotherapy.

techniques. ${ }^{7-11}$ Finally, to better define the role of IMRT in BC adjuvant RT, some phase III studies were performed. ${ }^{12-21}$

The aim of this review is to describe the available evidence coming from randomized trials on IMRT in BC.

\section{Methods}

We performed a study on PubMed about randomized trials on IMRT in BC. The search strategy was as follows: ("breast"[MeSH Terms] OR "breast"[All Fields]) AND IMRT[All Fields] AND ("random allocation"[MeSH Terms] OR ("random"[All Fields] AND "allocation"[All Fields]) OR "random allocation"[All Fields] OR "randomized"[All
Fields]). We included only randomized trials in English. We excluded prospective nonrandomized, retrospective, planning studies, reviews, letters, editorials, and case reports. Of the selected studies, we recorded the following data: authors, center, number of patients, selection criteria, median followup, dose and fractionation, target definition, study, planning, and delivery techniques in control and experimental arms, local control, survival, acute toxicity, late toxicity, and cosmetic results.

\section{Results \\ Search results}

After examining 30 potential papers based on title and abstract reviews, we selected 10 articles referring to 5 randomized studies reporting clinical results. In particular, 3 trials compared standard RT versus IMRT. ${ }^{12,14-17,20,21}$ One trial compared IMRT delivered in the supine versus the prone position. ${ }^{13}$ One trial compared standard RT versus accelerated partial breast IMRT. ${ }^{18,19}$ The primary end point of all these studies was the reduction of adverse events, and other reported end points were cosmesis, Patients Reported Outcomes Measures (PROMs), and QoL. One randomized trial was excluded because the authors reported only planning results ${ }^{22}$ impact of image guidance, ${ }^{23}$ and impact of the trial on clinical practice. ${ }^{24}$

\section{Study characteristics}

Donovan et $\mathrm{al}^{12}$ published in 2007 a comparison between a 2-dimensional wedged plan and either IMRT with the "step-and-shoot" technique or with a physical 3-dimensional compensator. They randomized 306 patients with "higher than average risk of normal tissue changes" based on breast size and shape. All were treated in the supine position with a dose of $50 \mathrm{~Gy}$ ( $2 \mathrm{~Gy} /$ fraction) to the whole breast plus 11.1 Gy (2.22 Gy/fraction) boost to the tumor bed. Results of the treatment were evaluated using photographic assessment performed at baseline and 1, 3, and 5 years from treatment and were scored with clinical assessment at the annual follow-up. They reported significantly fewer patients in the three dimensional (3D) IMRT group developing palpable induration but no significant differences in terms of breast discomfort, breast hardness, or QoL in either arm.

Mulliez et al ${ }^{13}$ published in 2013 the results of their phase III monocentric trial. They randomized 100 patients with large breast according to European cup size $\geq \mathrm{C}$ to supine 6 beam ( 3 medial and 3 lateral beams, nonopposing) IMRT irradiation versus 2 beam IMRT tangential fields in the prone position. The whole breast dose was 40.05 Gy (2.67 
Gy/fraction) plus a boost of $10 \mathrm{~Gy}$ (2.5 Gy/fraction). They recorded a significant reduction of G2-3 skin acute toxicity and improvement of dose homogeneity with reduced irradiation of heart and ipsilateral lung in patients treated in the prone position.

The Cambridge breast IMRT trial compared standard tangential techniques versus forward-planned IMRT. Eighthundred and fifteen patients out of 1,145 with significant dose inhomogeneity (more than $2 \mathrm{cc}$ of tissue receiving $>107 \%$ of the prescribed dose) were randomized. Patients were treated in the supine position with a dose of $40 \mathrm{~Gy}(2.67 \mathrm{~Gy} /$ fraction $)$ with $6 \mathrm{MV}$ photons prescribed to the ICRU50 reference point. Mixed energies ( 6 and $15 \mathrm{MV}$ ) were used, when required, in patients with larger separations. Nodal RT and a tumor bed boost were administered according to the local protocol. ${ }^{14-17}$

Results of the treatment were evaluated using both photographic and clinical assessment and patient-related questionnaires at 2 and 5 years after RT. The authors reported decreased skin telangiectasia and better cosmesis in the IMRT arm. However, no differences were recorded in terms of breast shrinkage, edema, indurations, and pigmentation..$^{14,16}$ The same group reported higher late toxicity risk in patients with larger breast volume and possibly smoking. ${ }^{15}$

From the same trial, the authors published the results in terms of PROMs. The study was unable to demonstrate the benefits of IMRT on PROMs (change in skin appearance, firmness to touch, reduction in breast size, and overall change in breast appearance since breast RT) at 5 years. Significant factors affecting PROMs were large breast, young age, baseline postsurgical cosmesis, and postoperative infections. ${ }^{17}$

Livi et al ${ }^{18}$ published in 2015 the 5 -year results of their phase III trial comparing standard whole breast (WB) radiotherapy ( 50 Gy in 25 fractions plus 10 Gy boost) with IMRTbased accelerated partial breast irradiation (APBI) (30 Gy in 5 fractions) in patients older than 40 years and tumor size less than $25 \mathrm{~mm}$. They recorded the same rate of ipsilateral breast recurrence $(1.5 \%)$ but lower acute and late toxicity and better cosmesis in patients receiving IMRT-APBI. Meattini et $\mathrm{al},{ }^{19}$ in the same year, published a subanalysis of the same trial including only patients older than 70 years. Even in this patient population, the authors did not record differences in terms of local recurrences but a reduced rate of acute and late toxicity in the IMRT-APBI treatment arm.

Pignol et $\mathrm{al}^{20}$ published in 2008 the results of a randomized trial on 331 patients comparing standard wedge-based RT with IMRT. They prescribed 50 Gy in 25 fractions to the clinical target volume (CTV; minimum dose: $95 \%$ ) plus 16 Gy boost. A significant improvement in terms of dose distribution was recorded in patients with IMRT. Furthermore, a significant reduction of moist desquamation was observed in the same patients. Moreover, smaller breast sizes (defined as bra sizes $32 \mathrm{~A}$ and $\mathrm{B}, 34 \mathrm{~A}$ and $\mathrm{B}$, and $36 \mathrm{~A}$ ) were associated with a decrease in moist desquamation. On the basis of these results, the authors concluded that largerbreasted women are most likely to benefit from IMRT. The same Canadian group published in 2016 the long-term outcomes of their multicenter randomized trial, reporting the results on 241 available patients. They did not observe significant differences between the two techniques in terms of chronic breast pain, cosmesis, and QoL. Chronic pain was documented as being associated only with young age and pain during RT, while subcutaneous fibrosis and telangiectasia were correlated with acute moist desquamation. Therefore, despite the evidence that breast IMRT improves the radiation dose distribution and reduces moist desquamation, the authors concluded that breast IMRT may not benefit all patients with regard to reduction of long-term side effects, but only patients presenting inhomogeneous dose distribution with standard RT technique. ${ }^{21}$

\section{Analysis of published studies}

The results from 5 randomized studies reporting clinical outcomes have been published in 10 papers. ${ }^{12-21}$ The characteristics and main results of these publications are reported in Table 1. Four trials were performed in Europe (all monocentric), ${ }^{12-19}$ and one multicentric trial was performed in Canada. ${ }^{20,21}$ The number of patients ranged between 100 and 850. Inclusion criteria were breast size and shape with $>$ risk of normal tissue change,${ }^{12}$ large breast according to European cup size $\geq \mathrm{C}$ in one study, ${ }^{13}>2 \mathrm{cc}$ receiving $>107 \%$ of the prescribed dose in another study, ${ }^{14-17}$ age $>40$ years and tumor $<25 \mathrm{~mm}$ in one study, ${ }^{18,19}$ and finally early stage with $\leq 3$ involved nodes in the last trial. ${ }^{20,21}$ Three studies compared standard WB RT with WB IMRT. ${ }^{12,14-17,20,21}$ The Florence study compared 3D-WB RT (50 Gy in 2 Gy/fractions + 10 Gy boost) with IMRT- APBI (30 Gy in 6 Gy/fraction). ${ }^{18,19}$ The Ghent trial compared supine multibeam IMRT of the breast with tangential IMRT in the prone position. ${ }^{13}$ Target definition was different in the analyzed trials, as reported in Table 1. Specifically, the definition of the target is not available in the two English studies. While in the Florence trial, the target was defined including $4 \mathrm{~mm}$ of the ipsilateral lung and excluding $3 \mathrm{~mm}$ from the skin, in the Ghent trial the target was defined as the whole breast, and in the Canadian trial $95 \%$ of the dose was delivered to CTV. These differences may have influenced the planning results but are unlikely to 


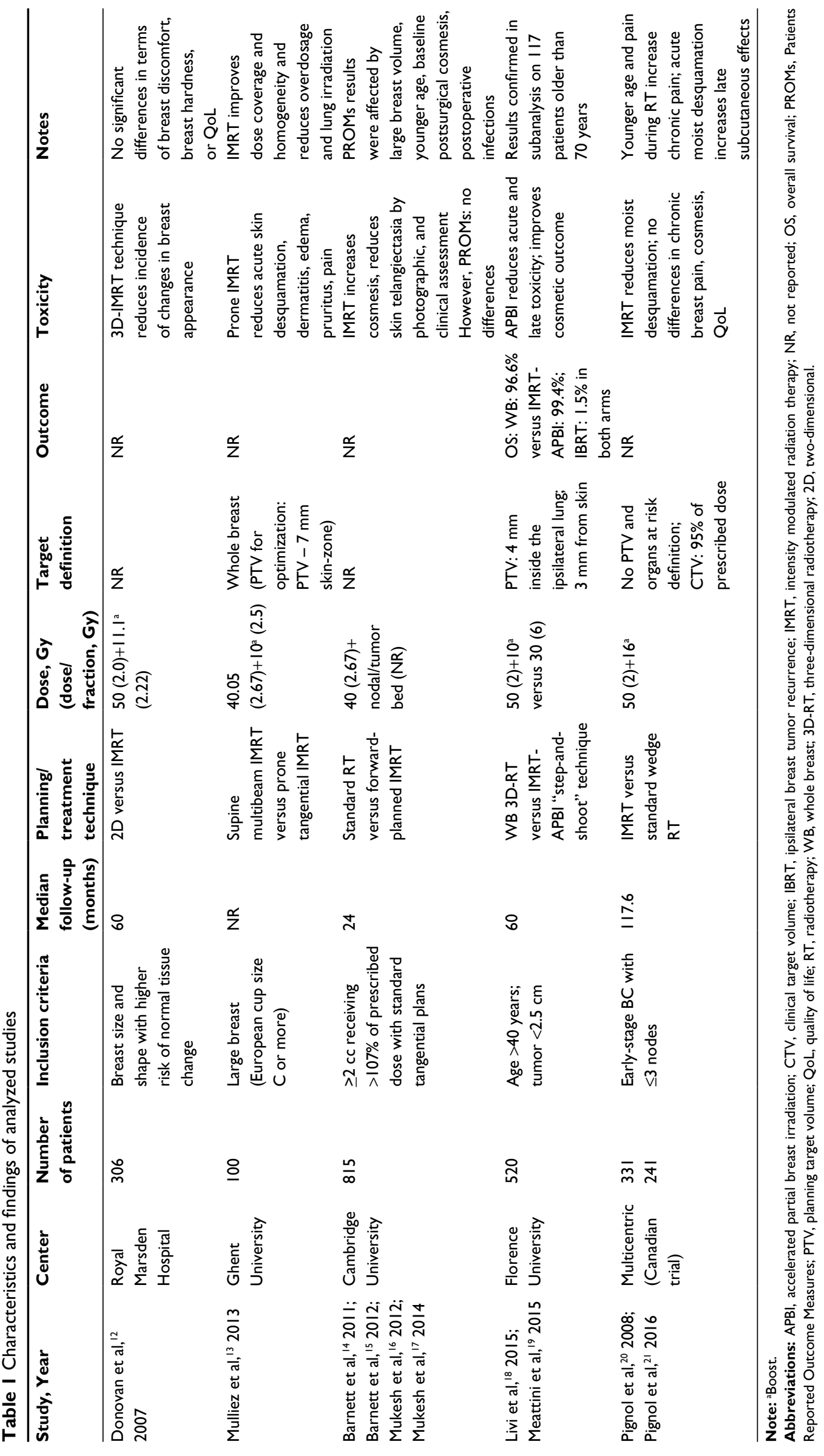


have affected the clinical comparison between IMRT and conventional techniques since target definition, except in the Florence study, was the same in both arms of the trials.

Acute toxicity was lowered as a result of using IMRT in all studies comparing IMRT with standard RT. ${ }^{13,18-21}$ Late toxicity was reduced in patients treated with IMRT in both Cambridge ${ }^{16}$ and Florence studies. ${ }^{18,19}$ The same studies ${ }^{14,16,18,19}$ reported improved cosmetic effects from using IMRT, while no differences in cosmesis were recorded in the Canadian trial. ${ }^{20,21}$ No differences were noted in terms of QoL in the Canadian trial ${ }^{20,21}$ and the Royal Marsden group, ${ }^{12}$ and in terms of PROMs in the Cambridge trial. ${ }^{17}$ Local control and overall survival showed no difference between standard and experimental arms in the only study reporting these outcomes. ${ }^{18,19}$

\section{Discussion}

We performed a review of literature on randomized controlled trials about IMRT in conservatively resected BC. We described 10 publications reporting data from 5 randomized trials describing clinical results outcomes. Some of these studies reported advantages in patients treated with IMRT mainly in terms of toxicity ${ }^{13,18-21}$ and partially in terms of cosmesis. ${ }^{12,14-19}$

These results confirm previous evidence coming mainly from retrospective cohort studies, ${ }^{8-10}$ one historically controlled trial, ${ }^{7}$ and one prospective controlled study. ${ }^{11}$ All these studies reported improved short-term tolerability in patients treated with IMRT. Furthermore, two of these studies reported improved long-term tolerability, always in patients treated with IMRT. ${ }^{9,10}$ Moreover, one study reported similar overall survival, disease-specific survival, freedom from ipsilateral breast tumor recurrence, distant metastasis, and secondary malignancies. ${ }^{10}$

However, a limitation of our review is that only three studies reported a comparison between IMRT and standard RT using the same targets and the same patients' position/ setup. In fact, the Florence study compared standard RT with IMRT-based APBI. Therefore, in this study, it is difficult to understand whether the recorded differences are related to the use of the IMRT technique alone. Furthermore, the Ghent study compared multibeam IMRT in supine position versus tangential IMRT in prone position. Finally, only one study included all patients treated with postoperative RT. ${ }^{20,21}$ In fact, the Cambridge study enrolled only patients with hotspot areas in the treated volume, while the Ghent and Royal Marsden trials enrolled only patients with large breast. ${ }^{12-17}$

These limitations obviously complicate the analysis of the trials' results. As we mentioned, one study reported reduced acute toxicity with IMRT but without comparing two arms of patients treated with the same target definition. ${ }^{18,19}$ Another study reported reduced toxicity in patients treated in the prone position compared with the supine position, and patients in both arms were treated with IMRT. ${ }^{13}$ The Cambridge group ${ }^{14,16}$ reported reduced skin telangiectasia in patients treated with IMRT, but we should note that patients enrolled in this trial had unfavorable anatomic characteristics in terms of dose homogeneity and conformity. Even the Florence group ${ }^{18,19}$ reported reduced late toxicity in IMRT-treated patients. Again, we should highlight that in the Florence study, the target was reduced in patients treated with IMRT. ${ }^{18,19}$ On the contrary, Pignol et al, ${ }^{21}$ after a 10 -year follow-up, reported no improved late toxicity in patients treated with IMRT probably because patients enrolled in both arms were treated in the same position, with the same dose, and using the same target definition. Furthermore, in this trial, patients were not selected on the basis of breast size and/or dose inhomogeneity. An improvement in terms of cosmesis was reported in these studies. ${ }^{14-19}$ The same considerations pertaining to late toxicity from these trials are obviously valid even in this issue. Again, we should note that in both the Canadian and the Royal Marsden trials, no differences were reported in terms of cosmesis.

Another limitation of our analysis is that it depends on whether the available evidence concerns mainly skin toxicity and not other potentially relevant adverse events such as pulmonary or cardiac toxicity and outcomes in terms of local control. However, in recent decades, as a matter of fact, the use of modern RT techniques has led to excellent outcomes in terms of disease control and reduction of toxicity other than skin damage. The almost exclusive focus on dermal toxicity is probably a result of this progressive improvement in clinical results.

Interestingly, only two studies included QoL or PROMs as end point. ${ }^{17,20,21}$ In both cases, no significant differences were recorded between patients treated with a standard versus the IMRT technique.

On the basis of the results of this review, we conclude that IMRT cannot be considered a standard treatment technique in unselected patients treated with postoperative RT for BC. In particular, this technique has no impact on patients' QoL. Therefore, its use could be reserved for patients with negative factors in terms of predicted treatment tolerability (large breast, postsurgical complication, and suboptimal dose distribution with large hot-spot volumes).

Further studies should be performed to develop predictive models of RT-induced toxicity allowing an improved identification of better candidates for this technique. 


\section{Conclusion}

In conclusion, on the basis of randomized trials, IMRT seems able to reduce toxicity in selected patients treated with postoperative RT for BC, particularly in combination with partial breast irradiation and prone patients positioning. Further analyses are needed to improve the selection of potential candidates for this treatment modality.

\section{Acknowledgments}

We sincerely thank Prof Mario Taffurelli and Dr Claudio Zamagni for reviewing and editing the manuscript.

\section{Disclosure}

The authors report no conflicts of interest in this work.

\section{References}

1. Ghoncheh M, Pournamdar Z, Salehiniya H. Incidence and mortality and epidemiology of breast cancer in the world. Asian Pac J Cancer Prev. 2016;17:43-46.

2. Overgaard M. Radiotherapy as part of a multidisciplinary treatment strategy in early breast cancer. Eur J Cancer. 2001;37(Suppl 7): S33-S43.

3. Hille-Betz U, Vaske B, Bremer M, et al. Late radiation side effects, cosmetic outcomes and pain in breast cancer patients after breastconserving surgery and three-dimensional conformal radiotherapy: risk-modifying factors. Strahlenther Onkol. 2016;192:8-16.

4. Moorthy S, Elhateer HS, Majumdar S, Mohammed S, Patnaik R; Narayanamurty. Dosimetric comparison of three dimensional conformal radiation therapy versus intensity modulated radiation therapy in accelerated partial breast irradiation. Indian J Cancer. 2016;53: $147-151$.

5. Wu S, Lai Y, He Z, et al. Dosimetric comparison of the simultaneous integrated boost in whole-breast irradiation after breast-conserving surgery: IMRT, IMRT plus an electron boost and VMAT. PLoS One. 2015;10:e120811.

6. Qiu JJ, Chang Z, Horton JK, Wu QR, Yoo S, Yin FF. Dosimetric comparison of 3D conformal, IMRT, and V-MAT techniques for accelerated partial-breast irradiation (APBI). Med Dosim. 2014;39:152-158.

7. Freedman GM, Anderson PR, Li J, et al. Intensity modulated radiation therapy (IMRT) decreases acute skin toxicity for women receiving radiation for breast cancer. Am J Clin Oncol. 2006;29:66-70.

8. Freedman GM, Li T, Nicolaou N, Chen Y, Ma CC, Anderson PR. Breast intensity-modulated radiation therapy reduces time spent with acute dermatitis for women of all breast sizes during radiation. Int $J$ Radiat Oncol Biol Phys. 2009;74:689-694.

9. Harsolia A, Kestin L, Grills I, et al. Intensity-modulated radiotherapy results in significant decrease in clinical toxicities compared with conventional wedge-based breast radiotherapy. Int J Radiat Oncol Biol Phys. 2007;68:1375-1380.
10. McDonald MW, Godette KD, Butker EK, Davis LW, Johnstone PA. Long-term outcomes of IMRT for breast cancer: a single-institution cohort analysis. Int J Radiat Oncol Biol Phys. 2008;72:1031-1040.

11. Morganti AG, Cilla S, Valentini V, et al. Phase I-II studies on accelerated IMRT in breast carcinoma: technical comparison and acute toxicity in 332 patients. Radiother Oncol. 2009;90:86-92.

12. Donovan E, Bleakley N, Denholm E, et al. Randomised trial of standard 2D radiotherapy (RT) versus intensity modulated radiotherapy (IMRT) in patients prescribed breast radiotherapy. Radiother Oncol. 2007;82:254-264.

13. Mulliez T, Veldeman L, van Greveling A, et al. Hypofractionated whole breast irradiation for patients with large breasts: a randomized trial comparing prone and supine positions. Radiother Oncol. 2013;108:203-208.

14. Barnett GC, Wilkinson JS, Moody AM, et al. The Cambridge Breast Intensity-modulated Radiotherapy Trial: patient- and treatmentrelated factors that influence late toxicity. Clin Oncol (R Coll Radiol). 2011;23:662-673.

15. Barnett GC, Wilkinson JS, Moody AM, et al. Randomized controlled trial of forward-planned intensity modulated radiotherapy for early breast cancer: interim results at 2 years. Int J Radiat Oncol Biol Phys. 2012;82:715-723.

16. Mukesh MB, Barnett G, Cumming J, et al. Association of breast tumour bed seroma with post-operative complications and late normal tissue toxicity: results from the Cambridge Breast IMRT trial. Eur J Surg Oncol. 2012;38:918-924.

17. Mukesh MB, Qian W, Wilkinson JS, et al. Patient reported outcome measures (PROMs) following forward planned field-in field IMRT: results from the Cambridge Breast IMRT trial. Radiother Oncol. 2014;111:270-275.

18. Livi L, Meattini I, Marrazzo L, et al. Accelerated partial breast irradiation using intensity-modulated radiotherapy versus whole breast irradiation: 5-year survival analysis of a phase 3 randomised controlled trial. Eur J Cancer. 2015;51:451-463.

19. Meattini I, Saieva C, Marrazzo L, et al. Accelerated partial breast irradiation using intensity-modulated radiotherapy technique compared to whole breast irradiation for patients aged 70 years or older: subgroup analysis from a randomized phase 3 trial. Breast Cancer Res Treat. 2015;153:539-547.

20. Pignol JP, Olivotto I, Rakovitch E, et al. A multicenter randomized trial of breast intensity-modulated radiation therapy to reduce acute radiation dermatitis. J Clin Oncol. 2008;26:2085-2092.

21. Pignol JP, Truong P, Rakovitch E, Sattler MG, Whelan TJ, Olivotto IA. Ten years results of the Canadian breast intensity modulated radiation therapy (IMRT) randomized controlled trial. Radiother Oncol. 2016;121:414-419.

22. Donovan EM, Ciurlionis L, Fairfoul J, et al. Planning with intensitymodulated radiotherapy and tomotherapy to modulate dose across breast to reflect recurrence risk (IMPORT High trial). Int J Radiat Oncol Biol Phys. 2011;79:1064-1072.

23. Donovan EM, Brooks C, Mitchell RA, et al; IMPORT Trials Management Group. The effect of image guidance on dose distributions in breast boost radiotherapy. Clin Oncol (R Coll Radiol). 2014;26:671-676.

24. Tsang Y, Ciurlionis L, Kirby AM, et al; IMPORT Trial Management Group. Clinical impact of IMPORT HIGH trial (CRUK/06/003) on breast radiotherapy practices in the United Kingdom. $\mathrm{Br} J$ Radiol. 2015;88:20150453.
Breast Cancer - Targets and Therapy

\section{Publish your work in this journal}

Breast Cancer - Targets and Therapy is an international, peerreviewed open access journal focusing on breast cancer research, identification of therapeutic targets and the optimal use of preventative and integrated treatment interventions to achieve improved outcomes, enhanced survival and quality of life for the cancer patient.

\section{Dovepress}

The manuscript management system is completely online and includes a very quick and fair peer-review system, which is all easy to use. Visit http://www.dovepress.com/testimonials.php to read real quotes from published authors. 